\title{
Supporting Information: Coupling of Ge Quantum Dots with Collective Sub-Radiant Modes of Silicon Nanopillar Arrays
}

Viktoriia Rutckaia, ${ }^{*} \dagger$ Frank Heyroth ${ }^{\ddagger}$ Georg Schmidt, Alexey Novikov,, ॥ Mikhail Shaleev, ${ }^{\S}$ Roman S. Savelev, ${ }^{\perp}$ Joerg Schilling, ${ }^{\dagger}$ and Mihail Petrov ${ }^{\perp}$ $\dagger$ Centre for Innovation Competence SiLi-nano ${ }^{\circledR}$, Martin-Luther-University Halle-Wittenberg, Karl-Freiherr-von-Fritsch-Str. 3, 06120 Halle (Saale), Germany $\ddagger$ Interdisciplinary center of material science, Martin-Luther-University Halle-Wittenberg, Heinrich-Damerow-Str. 4, 06120 Halle (Saale), Germany

\Institute of Physics, Martin-Luther-University Halle-Wittenberg, von-Danckelmann-Platz 3, 06120 Halle (Saale), Germany $\S$ Institute for Physics of Microstructures of the Russian Academy of Sciences, Academicheskaya Str. 7, Nizhny Novgorod 603950, Russia ||Lobachevsky University, Gagarin av. 23, Nizhny Novgorod 603950, Russia $\perp$ Department of Physics and Engineering, ITMO University, St. Petersburg 197101, Russia E-mail: viktoriia.rutckaia@physik.uni-halle.de 


\section{Polarization-resolved $\mu \mathrm{PL}$ from unprocessed layers}

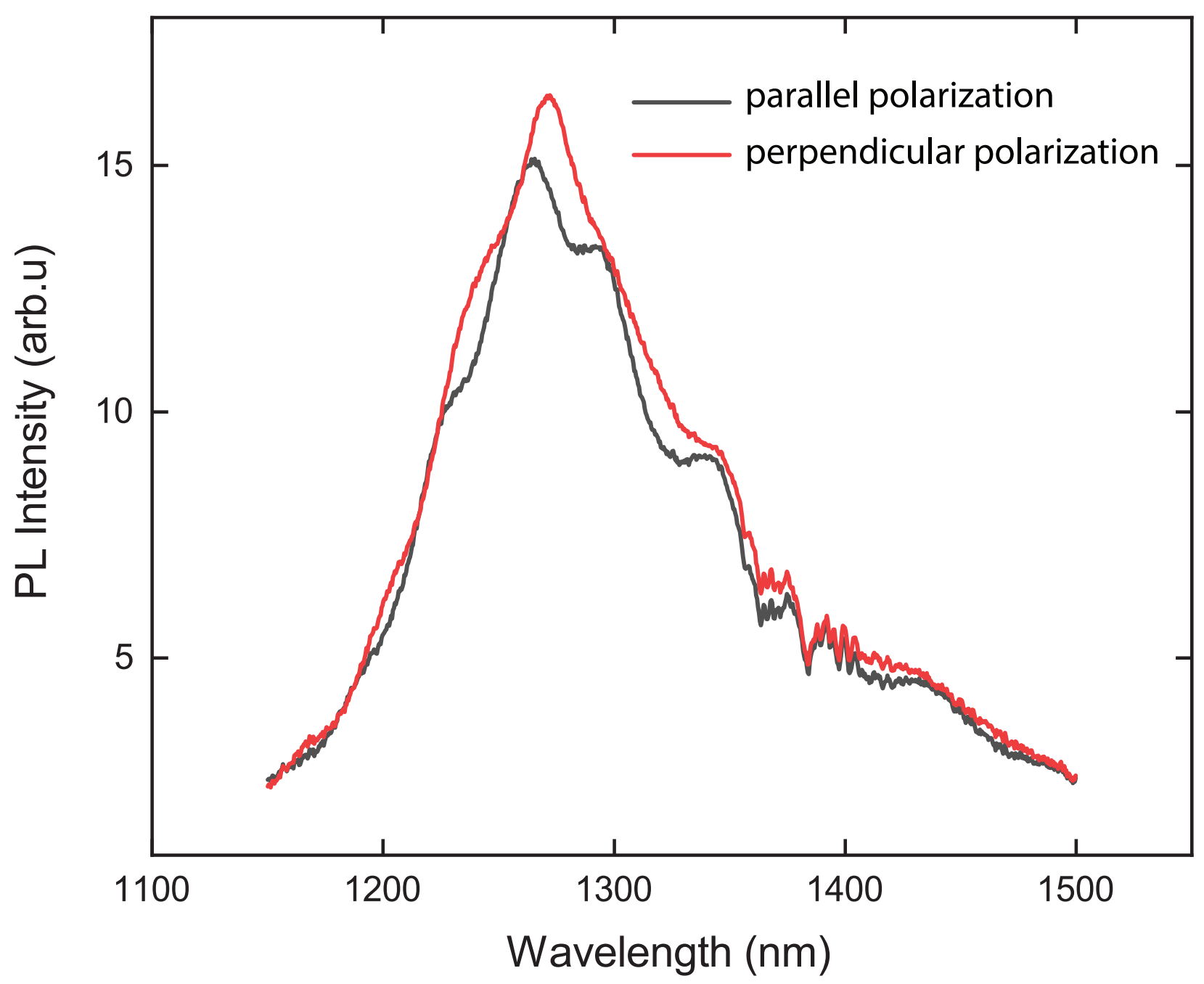

Figure 1: Polarisation-resolved $\mu \mathrm{PL}$ spectra of unprocessed layers of Si with Ge(Si) QDs.

Polarisation-resolved $\mu \mathrm{PL}$ of the unstructured substrate confirms that an ensemble of pristine QDs emits unpolarized light. Measurements were performed with the same conditions as for 11-element array shown in Fig. 1a) of the main manuscript. 


\section{PL enhancement in a single pillar and a chain}

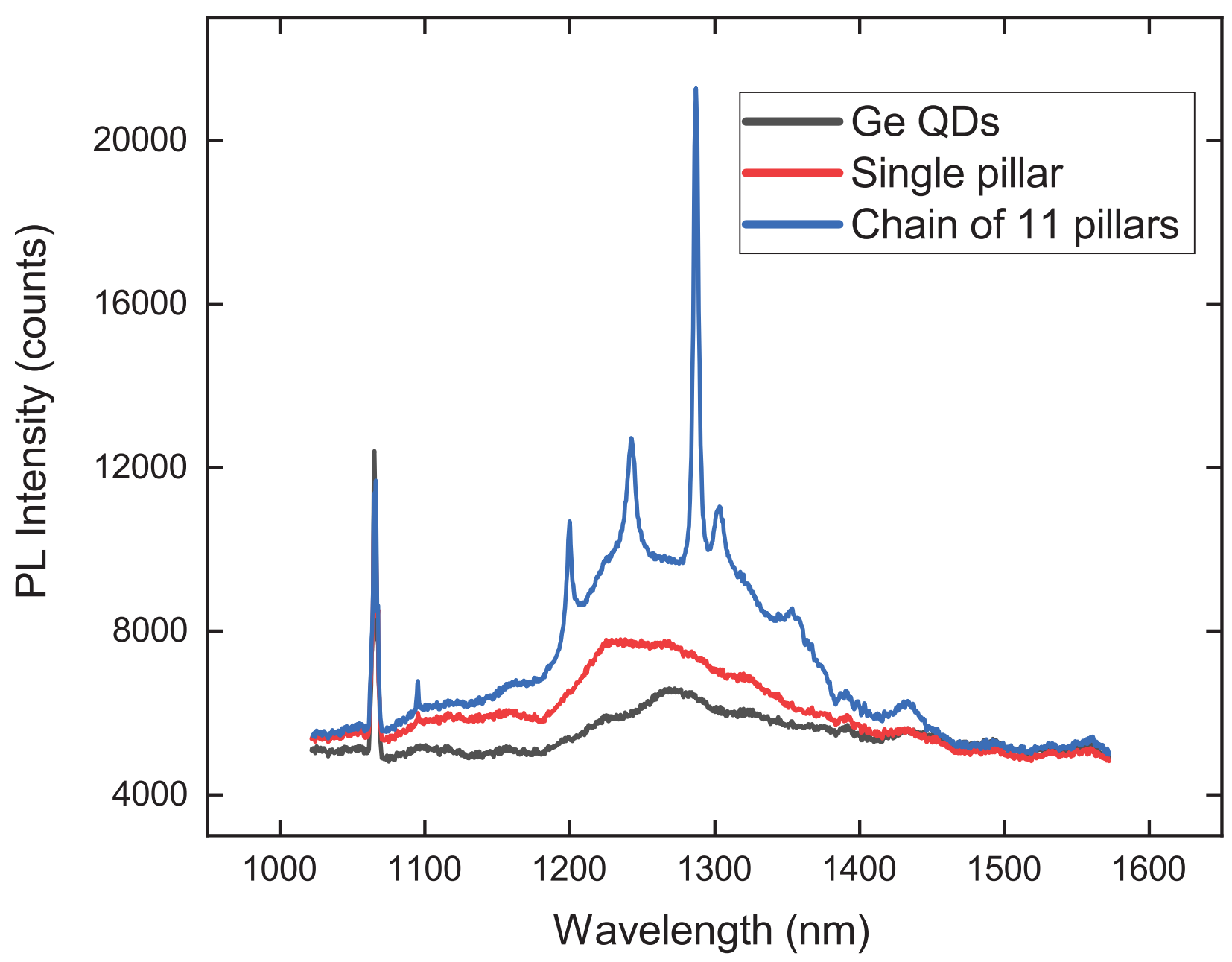

Figure 2: PL spectra of an unprocessed substrate with Ge(Si) QDS (black), single pillar (red) and a chain of 11 pillars (blue). A sharp peak at $1064 \mathrm{~nm}$ corresponds to the excitation laser second harmonic.

Direct comparison of the PL enhancement in various resonators is provided in Fig. 2. Here, PL spectra from an unprocessed substrate with Ge(Si) QDS (black), single pillar (red) and a chain of 11 pillars (blue) were measured in the same conditions. One can see a sharp peak at $1064 \mathrm{~nm}$ that corresponds to a second harmonic of the excitation laser and is not related to the sample PL. After background subtraction, PL enhancement of a single pillar and a chain relative to the signal from unprocessed QDs are 1.7x and 12x, respectively. 


\section{Eigenmode analysis of a single pillar}
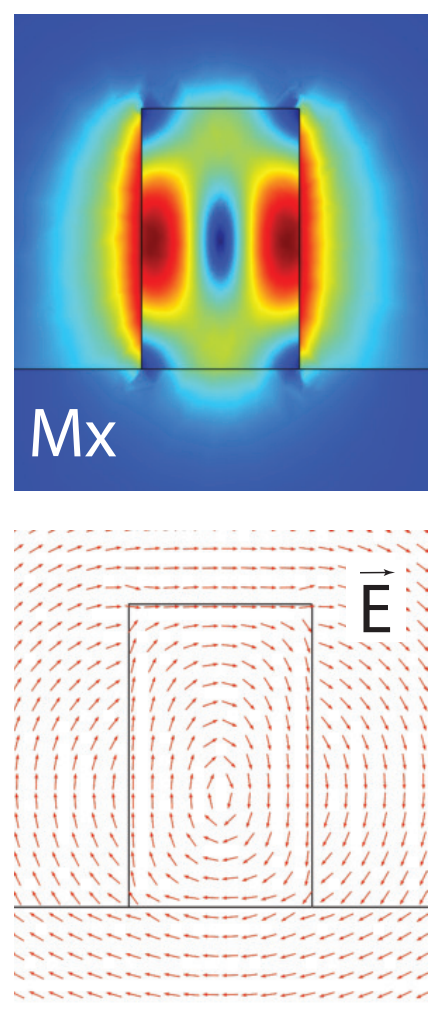
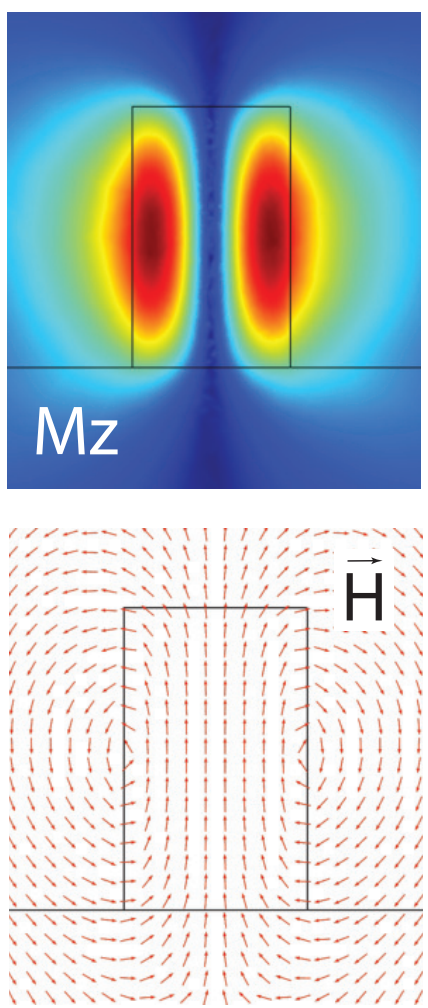

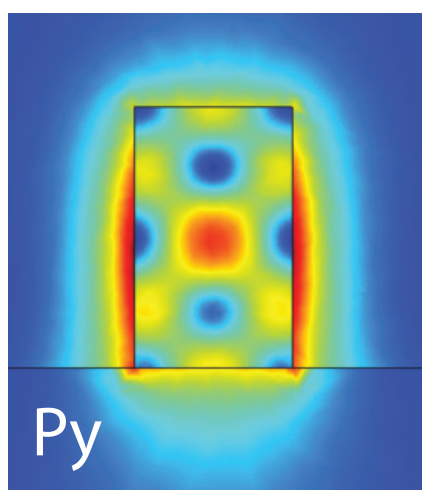

$\max$

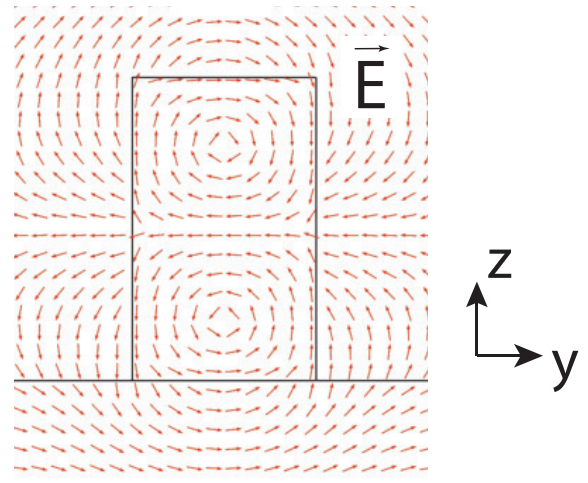

Figure 3: Top panel: simulated electric field intensity of $M_{x}, M_{z}$, and $P_{y}$ modes in the (xz)-plane. Bottom panel: corresponding electric and magnetic field lines.

Mode identification was performed based on the distribution of electric field currents and the dominant contribution in the multipole composition. Figure 3 shows electric field intensities and corresponding electric and magnetic field lines for the three modes considered in this work. One can see that $M_{x}$ mode has swirling electric filed in the $z y$-plane equivalent to an $x$-oriented magnetic dipole, hence the mode is called $M_{x}$. For the mode $M_{z}$, magnetic field lines have characteristic distribution of a $z$-oriented magnetic dipole. Similarly, $P_{y}$ mode has a distribution of electric field lines of a $y$-oriented electric dipole. Electric and magnetic dipole moments for single pillar eigenmodes are shown in Table 1 where mode labels coincide with the dominant multipole contribution. 
Table 1: Electric and magnetic dipole moments for single pillar eigenmodes.

Multipole components (1e-23 [C*m])

\begin{tabular}{|c|c|c|c|c|c|c|}
\hline Mode, freq & $\left|E D_{x}\right|$ & $\left|E D_{y}\right|$ & $\left|E D_{z}\right|$ & $\left|M D_{x}\right|$ & $\left|M D_{y}\right|$ & $\mid M D_{z}$ \\
\hline $2.13 \mathrm{E} 14+1$. & 3.1e-1 & $\begin{array}{l}1.45 \mathrm{e}-4 \\
\end{array}$ & 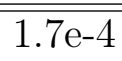 & $\overline{1.7 \mathrm{e}-3}$ & 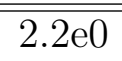 & $\overline{1.1 \mathrm{e}-}$ \\
\hline 2.14 & $3.9 \mathrm{e}-4$ & $3.2 \mathrm{e}-1$ & $4.7 \mathrm{e}-4$ & $3.9 \mathrm{e} 0$ & $8.3 \mathrm{e}-4$ & $4.8 \mathrm{e}-5$ \\
\hline 2.2 & $1.3 \mathrm{e}-4$ & $1.5 \mathrm{e}-4$ & $5.0 \mathrm{e}-5$ & $1.2 \mathrm{e}-4$ & $9.2 \mathrm{e}-5$ & $1.8 \mathrm{e} 0$ \\
\hline & $5 \mathrm{eC}$ & $9 \mathrm{e}-\frac{5}{5}$ & $.0 \mathrm{e}-4$ & $3.4 \mathrm{e}-4$ & $3.0 \mathrm{e}-1$ & $1.7 \mathrm{e}-4$ \\
\hline $2.52 \mathrm{E} 14+1$. & $2.1 \mathrm{e}-3$ & $2.6 \mathrm{e} 0$ & $2 \mathrm{e}-4$ & $4 \mathrm{e}-1$ & $3.8 \mathrm{e}-4$ & $.0 \mathrm{t}$ \\
\hline
\end{tabular}

\section{Parameters variation of the chain}

a)
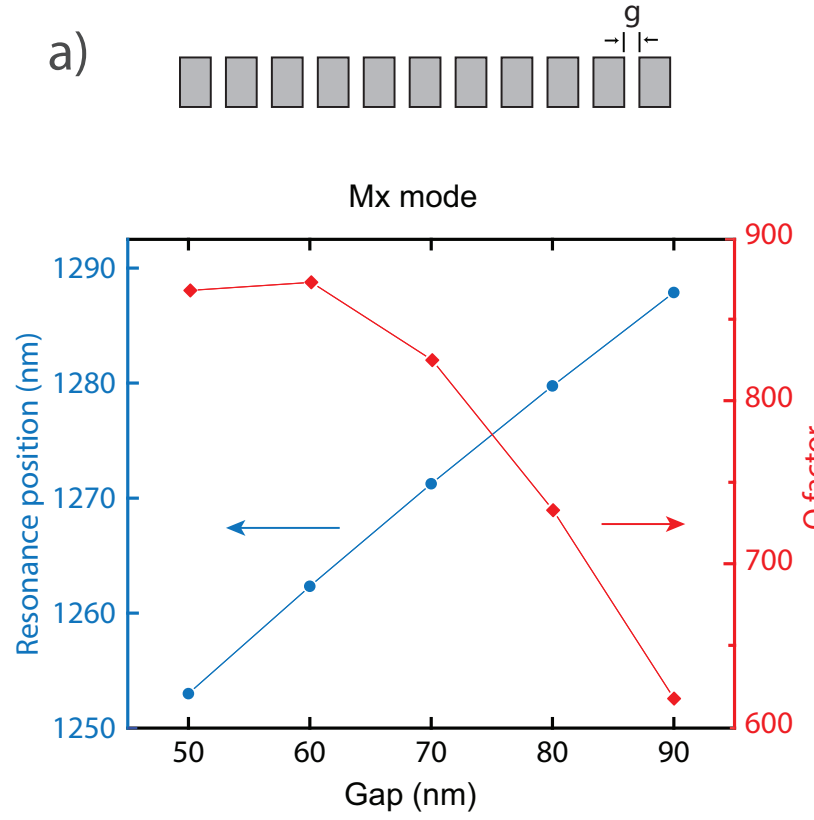

b)
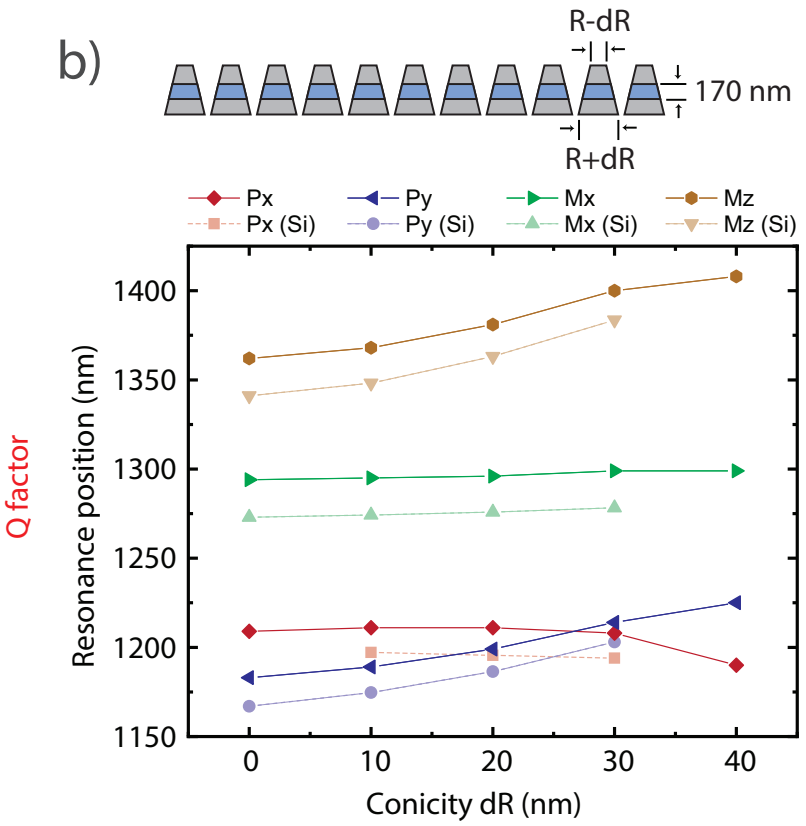

Figure 4: a) Calculated $M_{x}$ mode position (blue) and corresponding Q-factors (red) for the chain of $11 \mathrm{Si}$ pillars for various gaps between the pillars. b) Calculated dipole mode positions of the chain consisting of conical nanopillars with account for $\mathrm{Ge}(\mathrm{Si})$ QDs inclusions (solid lines) and for pure Si nanopillars (dashed lines). Inclusions of QDs are modeled as a $170 \mathrm{~nm}$ thick layer (marked with blue color in the figure) with effective refractive index corresponding to $\mathrm{Ge}(\mathrm{Si})$ QDs in $\mathrm{Si}$.

To elaborate more on the factors that can affect peak positions in the PL spectra shown in Fig. 2a) of the main manuscript, we performed calculations of 11-element chains taking into account the conical shape of the pillars, variation of the gap between the pillars, and local refractive index increase caused by the presence of $\mathrm{Ge}(\mathrm{Si})$ QDs. 
Figure 4a) shows calculated $M_{x}$ mode position and corresponding Q-factors of the chain of 11 pillars for various gaps between the pillars. One can see that the variation of the gap leads to the resonance shift of about $10 \mathrm{~nm}$ for all modes.

Figure 4b) shows calculated dipole mode positions of the chain consisting of conical nanopillars with account for $\mathrm{Ge}(\mathrm{Si})$ QDs inclusions (solid lines) and for pure Si nanopillars (dashed lines). Inclusions of QDs are modeled as a $170 \mathrm{~nm}$ thick layer (marked with blue color in the figure) with effective refractive index corresponding to $\mathrm{Ge}(\mathrm{Si}) \mathrm{QDs}$ in $\mathrm{Si}$. Ge dispersion is taken from Ref. ${ }^{1}$ One can see that the presence of QDs leads to a $\sim 20 \mathrm{~nm}$ shift of all resonances towards longer wavelengths. The conicity of the pillars, however, affect different modes differently. With the increase in the pillar wall inclination, $M_{z}$ and $P_{y}$ modes exhibit a red-shift, the $M_{x}$ mode stays at the same wavelength, and the $P_{x}$ mode is shifted to the shorter wavelength. The mixture of the above mentioned factors can explain the deviation of the calculated and measured resonance positions in Fig 2a) of the main manuscript.

\section{Spatial distribution of the collected PL signal}

Though the near-field energy is localized in the middle of the array, the radiation of the electromagnetic energy occurs from the edges of the structures as it can be considered as a defect in infinite waveguiding structure. This can be evidently seen in the simulation results provided in Fig. 5 a)-d) for an 11-particle chain, where the numerically computed field intensity of the resonant $M_{x}$ mode is plotted in planes parallel to the interface plane at different distances $\Delta$ from the array $(0,200,1000,2000 \mathrm{~nm})$. One can see that near the array, the energy is mainly localized in the middle of the chain, but already at a distance a $200 \mathrm{~nm}$ and further, there appear intense regions corresponding to the edges of the array.

We have evidenced it in mapping $\mu \mathrm{PL}$ experiment. Fig. 5e) shows the schematic of the mapping experiment where the laser light coming through the objective lens placed above 

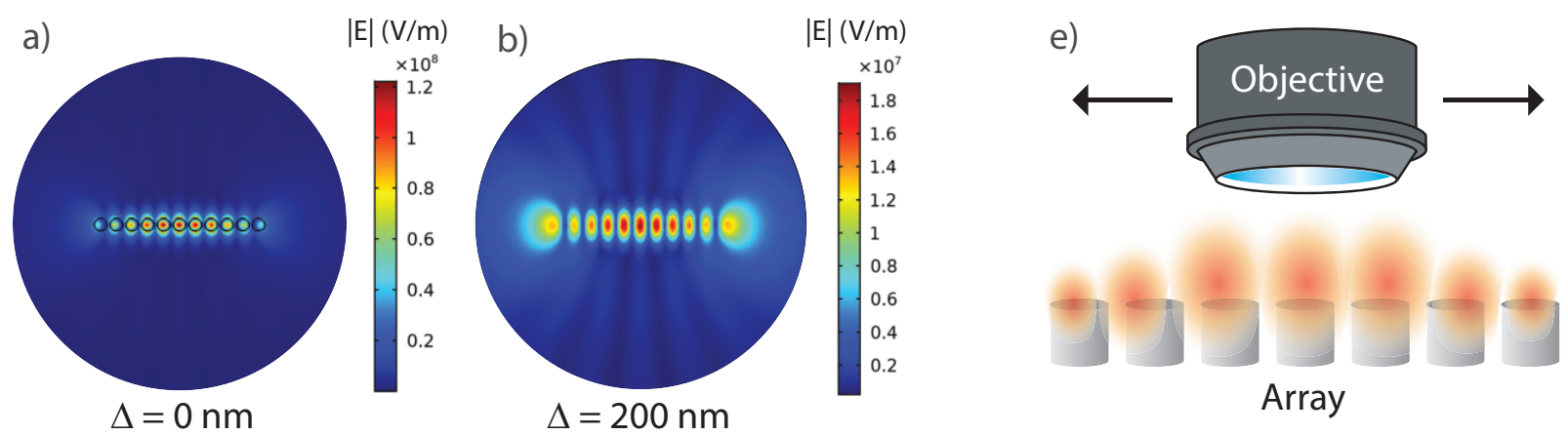

|E $(\mathrm{V} / \mathrm{m})$
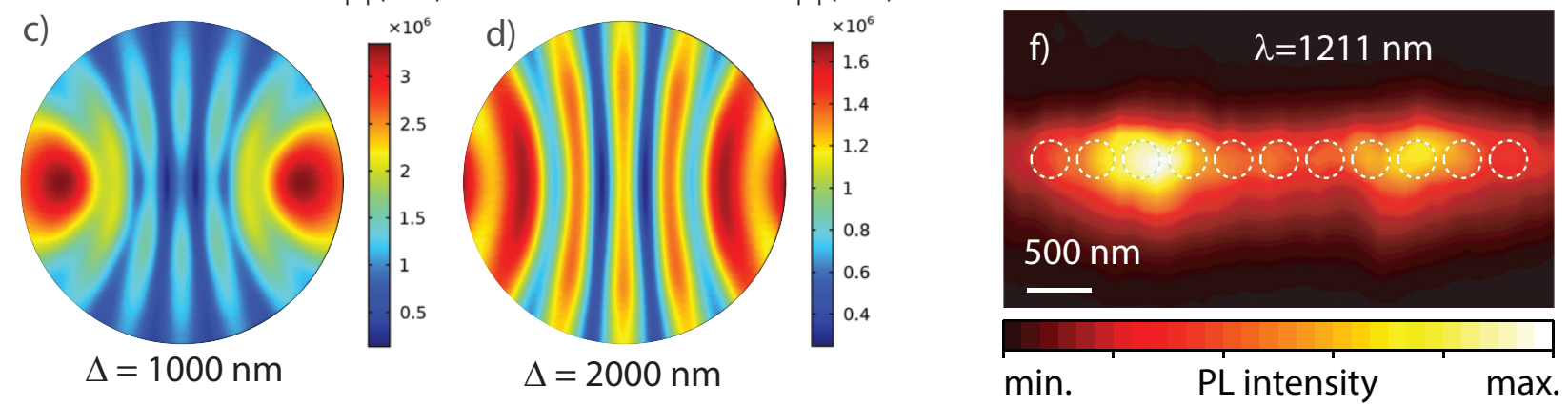

Figure 5: a)-d) Distribution of the electric field intensity of the 11-pillar chain at $M_{x}$ resonance for various distances $\Delta=0,200,1000,2000 \mathrm{~nm}$ from the surface of the chain. e) Schematic of the mapping experiment where the light emission is collected above the chain. The mapping spatial resolution is $100 \mathrm{~nm}$, laser spot size is about $1.5 \mu \mathrm{m}$. f) $\mu \mathrm{PL}$ map of the 11-pillars chain (outlined with dashed circles) measured at $1211 \mathrm{~nm}$. PL intensity maxima positions indicate the trade-off between preferable in-plane radiation direction and the collection efficiency of the objective lens, which decreases at the edges of the chain.

the structure can be spatially positioned on different locations of the sample (Duo Scan mode of Horiba LabRAM $\mu$ PL setup). Mapping spatial resolution is $100 \mathrm{~nm}$, laser spot size is about $1.5 \mu \mathrm{m}$. For each position of the excitation source, light emission spectra is recorded as explained in the main manuscript. The obtained PL-map for an 11 pillars chain (pillar positions are outlined with dashed circles) at $1211 \mathrm{~nm}\left(P_{y}\right.$ mode) is shown in figure $5 \mathrm{f}$ ). Other pronounced resonances $\left(P_{x}, M_{x}\right)$ qualitatively have same PL distributions. One can see that the intensity is larger at the sides of the sample, however not at the very edges as it is shown in numerical simulations. The reason is that at the very edges the overlap of the excitation beam with the structure is not maximal, thus, maximal signal is detected when the convolution of excitation and collection efficiency is maximal. 


\section{Polarization-resolved measurements}
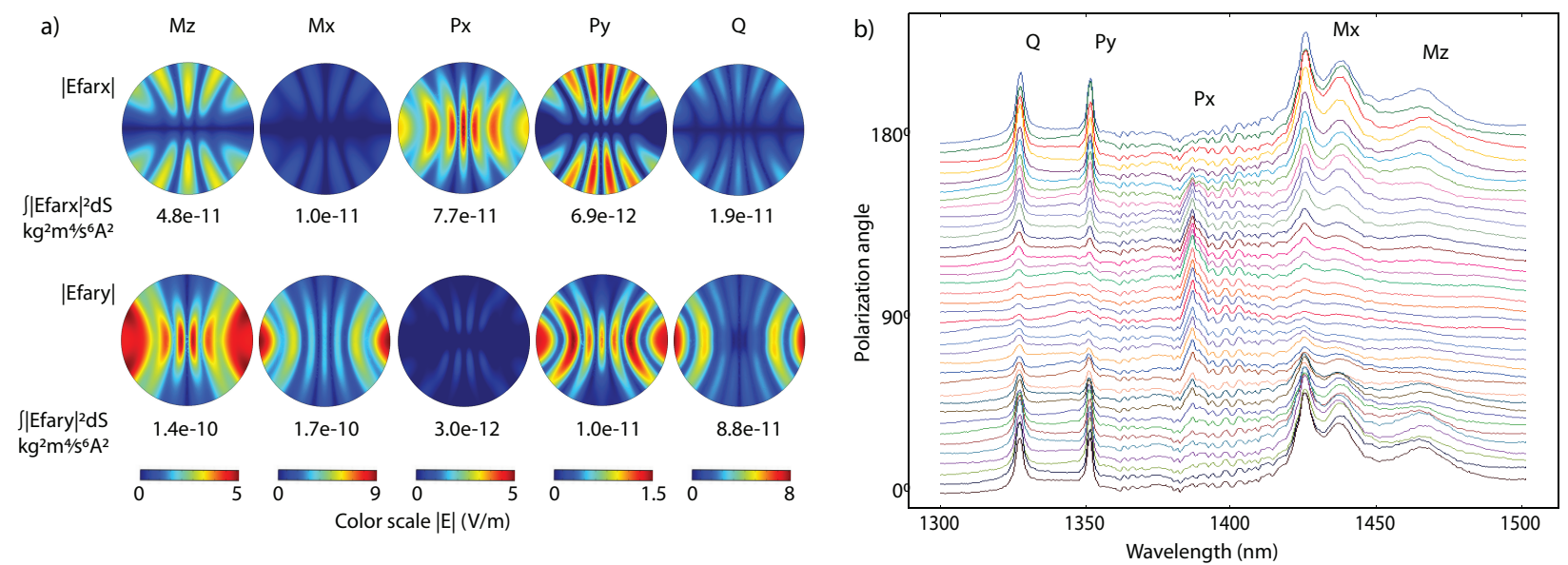

Figure 6: a) Calculated distribution of the far-field components (top panel $E_{\text {far } x}$, bottom panel $\left.E_{\text {fary }}\right)$ for collective modes of a chain of 11 pillars with the height $600 \mathrm{~nm}$, pillar diameter $300 \mathrm{~nm}$, gap $70 \mathrm{~nm}$. The field is plotted in a spatial angle corresponding to the $N A=0.9$. For each mode, the scale of the color map is depicted on the bottom of the Figure. Below each map, an integral of the far-field component over the spatial angle is provided. b) Polarization-resolved PL spectra of a chain of 11 pillars of the height $600 \mathrm{~nm}$, pillar diameter $300 \mathrm{~nm}$, gap $70 \mathrm{~nm}$. From bottom to the top an analyzer was rotated by 5 degrees. Peaks are marked according to the calculated polarization of the chain modes.

To address the inheritance of the polarization in near and far-fields, we performed additional simulations. In Figure 6 a), we plot a distribution of electric far field components for collective modes of an 11-mer in a plane $1350 \mathrm{~nm}$ above the chain, which corresponds to the $N A=0.9$. Top panel shows $E_{\text {farx }}$ component of the electric field oriented along the chain, bottom panel shows $E_{\text {fary }}$ oriented perpendicular to the chain. The color map scale for each mode is the same for $x$ - and $y$ - components and therefore one can compare their intensities. One can see that $M_{z}, M_{x}, P_{y}$, and $Q$ (quadrupole) modes have a dominating contribution to the electric field intensity from the $y$-component resulting in a $y$-polarized PL signal while the $P_{x}$ mode has a dominating contribution from the $x$-component. We also provide calculated values of the integral of the squared electric far field over the plotted surface that are noted below each color map. To elaborate a bit more about our strategy, we provide a polarization-resolved PL measurement results for a modeled chain of 11 pillars in Figure $6 \mathrm{~b}$ ). The bottom spectrum was measured with an analyzer oriented perpendicular 
to the chain ( $y$-polarization), and for each spectrum stacked above the analyzer was rotated by 5 degrees. One can see four peaks with $y$-polarization and one peak with orthogonal $x$-polarization. This agrees with our simulated far-field polarization for $M_{z}(y-), M_{x}(y-)$, $P_{x}(x-), P_{y}(y-)$, and $Q(y-)$ modes.

\section{References}

(1) Nunley, T. N.; Fernando, N. S.; Samarasingha, N.; Moya, J. M.; Nelson, C. M.; Medina, A. A.; Zollner, S. Optical constants of germanium and thermally grown germanium dioxide from 0.5 to $6.6 \mathrm{eV}$ via a multisample ellipsometry investigation. Journal of Vacuum Science $\&$ Technology B 2016, 34, 061205. 